ACCEPTED MANUSCRIPT

\title{
BMP-2 functionalized PEDOT:PSS-based OECTs for stem cell osteogenic differentiation monitoring.
}

To cite this article before publication: Francesco Decataldo et al 2019 Flex. Print. Electron. in press https://doi.org/10.1088/2058-8585/ab5bfc

\section{Manuscript version: Accepted Manuscript}

Accepted Manuscript is "the version of the article accepted for publication including all changes made as a result of the peer review process, and which may also include the addition to the article by IOP Publishing of a header, an article ID, a cover sheet and/or an 'Accepted Manuscript' watermark, but excluding any other editing, typesetting or other changes made by IOP Publishing and/or its licensors"

This Accepted Manuscript is @ 2019 IOP Publishing Ltd.

During the embargo period (the 12 month period from the publication of the Version of Record of this article), the Accepted Manuscript is fully protected by copyright and cannot be reused or reposted elsewhere.

As the Version of Record of this article is going to be / has been published on a subscription basis, this Accepted Manuscript is available for reuse under a CC BY-NC-ND 3.0 licence after the 12 month embargo period.

After the embargo period, everyone is permitted to use copy and redistribute this article for non-commercial purposes only, provided that they adhere to all the terms of the licence https://creativecommons.org/licences/by-nc-nd/3.0

Although reasonable endeavours have been taken to obtain all necessary permissions from third parties to include their copyrighted content within this article, their full citation and copyright line may not be present in this Accepted Manuscript version. Before using any content from this article, please refer to the Version of Record on IOPscience once published for full citation and copyright details, as permissions will likely be required. All third party content is fully copyright protected, unless specifically stated otherwise in the figure caption in the Version of Record.

View the article online for updates and enhancements. 


\title{
BMP-2 functionalized PEDOT:PSS- based OECTs for stem cell osteogenic differentiation monitoring.
}

\author{
Francesco Decataldo ${ }^{1,2}$, Victor Druet ${ }^{1,3}$, Anna-Maria Pappa ${ }^{1}$, Ellasia Tan ${ }^{4}$, Achilleas \\ Savva $^{1,3}$, Charalampos Pitsalidis ${ }^{1}$, Sahika Inal ${ }^{3}$, Ji-Seon Kim ${ }^{4}$, Beatrice Fraboni ${ }^{2, *}$, \\ Róisín M. Owens ${ }^{1, *}$ and Donata landolo ${ }^{1,5, *}$. \\ ${ }^{1}$ Department of Chemical Engineering and Biotechnology, University of Cambridge, Cambridge, United \\ Kingdom \\ ${ }^{2}$ Department of Physics and Astronomy, University of Bologna, Bologna, Italy. \\ ${ }^{3}$ Biological and Environmental Science and Engineering Division, King Abdullah University of Science \\ and Technology, Thuwal, Saudi Arabia \\ ${ }^{4}$ Department of Physics and Centre for Plastic Electronics, Imperial College London, London, United \\ Kingdom. \\ ${ }^{5}$ Mines Saint-Etienne, University of Lyon, Université Jean Monnet, INSERM, Saint-Etienne France. \\ E-mail: B.F.: beatrice.fraboni@unibo.it, R.M.O.: rmo37@cam.ac.uk, D.I.: donata.iandolo@emse.fr
}

Received xxxxxx

Accepted for publication $\operatorname{xxxxx}$

Published xxxxxx

\begin{abstract}
Stem cell osteogenic differentiation is a complex process, associated with a number of events such as the secretion of collagen type I, osteopontin, osteonectin, osteocalcin and Bone Morphogenic Protein 2 (BMP-2). These molecules can be used as markers to monitor stem cell fate while studying the effects of a specific osteogenic differentiation treatment (e.g. electrical stimulation). Currently available techniques, such as the evaluation of the expression levels of specific genes and end-point biochemical assays, do not allow real-time monitoring of cellular processes, therefore overlooking potentially interesting information. This study explores a promising functionalization strategy towards on-line electrical monitoring of stem cell osteogenic differentiation process, using an organic electrochemical transistor (OECT) to detect cytokines of interest, secreted by cells during the osteogenic differentiation process, such as BMP-2. In this work, antibodies against BMP-2 were anchored on the poly(3,4-ethylenedioxythiophene):polystyrene (PEDOT:PSS) sulfonate gate electrode of an OECT. The biofunctionalization process was evaluated using multiple techniques such as Atomic Force Microscopy, Electrochemical Raman Spectroscopy, Quartz crystal microbalance. Electrode properties were assessed by running chronoamperometric studies, as well as by characterizing the PEDOT:PSS thin film resistance to ion flow by electrochemical impedance spectroscopy and OECT performance using transient (AC) measurements. Finally, a proof-of-concept, biosensor measurement was performed to test our functionalization strategy for sensing, proving that the antibody-functionalized OECTs were able to detect recombinant BMP-2 at levels that are comparable to those used for in vitro stimulation of bone regeneration via soluble osteoinductive factors.
\end{abstract}

Keywords: biosensor, OECT, stem cell, bioelectronics, in vitro models, PEDOT:PSS

\section{Introduction}

59

60
Globally, the risk of an osteoporotic fracture affects one in three women and one in five men over the age of fifty.[1] Increasing life expectancy may be seen as a direct result of 
improved health conditions. However, it imposes an important economic burden on the healthcare system. In Europe alone, the annual cost of osteoporotic fractures is estimated to pass $€ 76$ billion by 2050 . Osteoporosis is a musculoskeletal disorder accompanied by a progressive decrease in bone mechanical resistance, eventually leading to an increased incidence of fractures.[2,3] Among the different approaches that have been established to treat this condition, the use of stem cells is highly relevant and has seen a widespread use lately.[4,5] Several approaches have been put in place to direct stem cell differentiation towards bone forming cells,[6] with the use of soluble factors to guide cell fate being the gold standard for in vitro differentiation. Indeed, relevant factors (e.g. dexamethasone, a source of phosphate, and vitamin C) are added to the culture medium to imbalance cell fate towards the osteogenic pathway.[7,8] Alternative strategies leverage the potential offered by material science and therefore use mechanical and geometrical cues as well as surface functionalization, to influence cell behavior and commitment to a specific lineage. $[9,10]$ Lately, innovative approaches see the use of smart materials and physical stimuli to direct stem cell fate.[11],[8],[12] The process of stem cell differentiation is a complex and articulate chain of events accompanied by the secretion of collagen type I and cytokines such as osteocalcin, bone sialoprotein and osteopontin, amongst others.[13,14] Influencing cell fate by external stimulation is a powerful tool to implement innovative solutions, possibly leading to clinically relevant approaches.[15,16] The strategies used to monitor cell differentiation and cell commitment to a specific cell lineage usually involve endpoint enzymatic assays (e.g. Alkaline phosphatase activity), ELISA assays and gene expression profiling. Being able to run real-time monitoring of cellular differentiation processes would allow researchers to acquire new data and helpful information, potentially overlooked by standard methods such as end-point assays. Indeed, a new trend in the field of organson-chips and in vitro tissue models is to harness the potential offered by the integration of sensing units with the culture chambers to continuously monitor the system. [17-19]

The present work falls in this area as we have developed a strategy for a sensing device for stem cell differentiation monitoring, with the potential to be integrated within a cell culture chamber. Our technology is based on the coupling of the electrochemical properties of poly $(3,4-$ ethylenedioxythiophene): polystyrene sulfonate (PEDOT:PSS), and the specificity brought by monoclonal antibodies targeting the analyte of choice. PEDOT:PSS is a conducting polymer with the ability to conduct both ionic and electronic currents owing to its composition, consisting of a conjugated polymer, PEDOT, and the polyanion, PSS, which provides the sulphate counterions that compensate for the holes travelling along the conjugated backbone.[20] This property contributes to making PEDOT:PSS one of the materials of choice at the interface with biological systems.[18,21] Several devices have been developed based on this conducting polymer for a number of diverse applications embracing different fields such as tissue engineering, neural recording, neuromorphic computing, wearable and in vitro sensing. [22][23],[11], [24,25],[26-28] These materials hold promise for the electrical stimulation of osteogenic differentiation together with other electroactive materials and composite that have been explored for chronic electrical stimulation.[29,30] PEDOT:PSS-based organic electrochemical transistors (OECTs) are highly suited to sensing applications in aqueous environments, due to their ability to translate a biological event (e.g. the binding of an antibody on their surfaces) into an electronic readout. OECTs are three terminal devices, with a conducting polymer channel, and a (metallic or polymeric) gate electrode, connected through an electrolyte solution. The conductivity of the PEDOT:PSS channel, thus the electronic output current, can be modulated by the potential applied at the gate electrode, which, in turn, pushes ions into the channel of the device, changing its conductivity. As a result of bulk interactions of electrolyte ions and the charges in the channel, these devices offer significant amplification in ion-to-electron transduction. [31,32] Functionalization of the channel or gate with appropriate bío-recognition elements has been shown to yield biosensors for a variety of analytes. [33,34] In previous works, researchers used gold nanoparticles or enzymatic reactions to amplify the signal connected to the binding event or to detect the target analyte tout-court.[33,35,36] In the present study, instead, we developed a functionalization strategy where no charged species are harnessed to amplify the signal and that could be deployed in a sensing device for the specific detection of molecules released during stem cell osteogenic differentiation. We carried out proof of concept tests using BMP-2 as a testbed for our technology. This approach holds promise for the future development of devices to be integrated within bioreactors to monitor the effect of the applied stimulation protocol for stem cell differentiation and therefore have better insights into cellular mechanisms.

\section{Experimental}

\subsection{Material and Chemicals. PEDOT:PSS Clevios PH1000} (PEDOT:PSS) was purchased from Heraeus. 3glycidoxypropyltrimethoxysilane (GOPS), ethylene glycol (EG), 4-dodecylbenzenesulfonic acid (DBSA) and (3Aminopropyl)triethoxysilane (APTES) were obtained from Sigma-Aldrich. Tween ${ }^{\circledR} 20$ was bought from Fisher BioReagents and DyLightTM 405 Streptavidin (SA) from Thermo Scientific. Sulfo-NHS-Biotin, Recombinant AntiBMP2 antibody [EPR20807] (ab225898) (Rabbit recombinant monoclonal BMP-2 antibody), (FITC)-Anti-BMP2 antibody (ab136177) (Rabbit, polyclonal BMP-2 antibody) and 
Recombinant human BMP-2 protein (Active) (ab50099) were purchased from Abcam (UK).

\subsection{Electrodes deposition and functionalization}

\subsubsection{PEDOT:PSS thin film deposition. $10 \% \mathrm{w} / \mathrm{w}$ of} ethylene glycol (EG), $1 \% \quad \mathrm{w} / \mathrm{w}$ of 3glycidoxypropyltrimethoxysilane (GOPS), and $0.5 \% \mathrm{w} / \mathrm{w}$ of 4-dodecylbenzenesulfonicacid (DBSA) were added to the PEDOT:PSS suspension and ultrasonication was used after each addition to improve the additive dispersion. The suspension was then filtered using $0.45 \mu \mathrm{m}$ hydrophilic Minisart NML Sartorius filters.

Glass substrates $\left(25 \times 26 \mathrm{~mm}^{2}\right)$ were cleaned by sonication in subsequent baths of distilled water, acetone and isopropanol. The cleaned substrates were plasma treated (100W, 5min, Air) and a thin film $(210 \pm 20 \mathrm{~nm})$ of PEDOT:PSS was deposited by spin coating at 1500 RPM for $30 \mathrm{~s}$ with $3 \mathrm{~s}$ of acceleration. Samples were subsequently thermally treated at $120^{\circ}$ for $1 \mathrm{~h}$.

2.2.2 APTES Deposition. Two methods were explored to functionalize the PEDOT:PSS surface with an APTES layer, namely chemical vapor deposition and a soaking step.

APTES deposition by chemical vapor deposition (CVDAPTES). PEDOT:PSS films were plasma treated (20W, $1 \mathrm{~min}$, in air) to introduce hydroxyl groups therefore facilitating APTES binding. $500 \mu \mathrm{L}$ of APTES were placed in a thermally conductive container with the target sample facing down inside a desiccator chamber. The volume of the vacuum jar is approximatively $2.3 \mathrm{~L}$. The pressure was first decreased down to $\sim 35 \mathrm{mbar}$, then the hot plate was set to $140{ }^{\circ} \mathrm{C}$. The CVD was stopped after 2 hours; the base of the vacuum bell reached the temperature of $65^{\circ} \mathrm{C}$. Exhaust APTES vapor was then expelled and few drops of distilled water were deposited on the heating substrate, keeping the samples inside the vacuum bell for $1 \mathrm{~h}$ to allow the crosslinking of APTES molecules. [37]

APTES deposition by soaking (S-APTES). To perform APTES deposition by soaking, PEDOT:PSS films were plasma treated as reported above and then immersed in a $2 \%$ v/v APTES solution in a 95\% ethanol aqueous solution for 19 hours. Then, substrates were rinsed carefully with ethanol and distilled water, then dried with a nitrogen flux. Subsequently, the annealing $\left(65^{\circ} \mathrm{C}\right.$ for 5 hours) was run to crosslink the APTES molecules.

2.2.3 Biotin Functionalization. APTES-functionalized substrates were immersed in a $4 \mathrm{mg} / \mathrm{mL}$ biotin solution for 1 hour. Afterwards, they were rinsed carefully with distilled water and dried with a nitrogen flux.

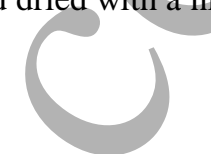

2.2.4 Streptavidin Functionalization. After biotin deposition, samples were incubated in a solution of 200 $\mu \mathrm{g} / \mathrm{mL}$ streptavidin in $1 \mathrm{X}$ Phosphate Buffer Saline (no Calcium no Potassium, Sigma Aldrich) for 1 hour. Substrates were subsequently rinsed four times alternating a $0.05 \%$ solution of Tween ${ }^{\circledR} 20$ in PBS (PBST) and PBS. These washing steps were done under constant agitation at $100 \mathrm{rpm}$ to remove aspecific binding.

\subsubsection{Antibodies Functionalization. Anti BMP-2 antibodies} (Abcam, UK) were biotinylated with a biotinylation kit (ab201796 -Biotin, Type B, Fast Conjugation Kit, Abcam, UK) following the manufacturer's instructions to obtain a 0.83 $\mu \mathrm{g} / \mathrm{ml}$ solution. Streptavidin-functionalized samples were incubated with the as-prepared solution for $1 \mathrm{~h}$, rinsed with PBS and Milli-Q water and then dried with nitrogen flux. Samples were stored in $1 \mathrm{X}$ PBS.

2.2.6 BMP-2 Functionalization. Finally, samples were incubated with $15 \mu \mathrm{l}$ of $0.0016 \mu \mathrm{g} / \mu \mathrm{l}$ BMP-2 (Abcam, UK) in PBS for 1h, rinsed with PBS and Milli-Q water and then dried with a nitrogen flux. Samples were stored in $1 \mathrm{X}$ PBS.

\subsection{Characterization techniques.}

2.3.1 Contact Angle. Static contact angle measurements were run using a Contact Angle System OCA (Dataphysics) dispensing a $5 \mu \mathrm{L}$ drop of deionized water onto the surface of the sample. At least five values of contact angle per sample were obtained and three different samples were measured.

2.3.2 Atomic Force Microscopy. A Veeco Dimension 3100 Scanning Probe System was used for Atomic Force Microscopy in non-contact (NSC15 tip) and conductive (MLCT tip) mode, for morphology and conductivity studies on the surface, respectively.

2.3.3 Chronoamperometry. The electrochemical cell comprised of an $\mathrm{Ag}$ wire reference electrode, Pt-coated fluorine doped tin oxide (FTO) counter electrode, working FTO electrode with sample deposited as a thin film and diluted 0.01X PBS as the electrolyte. The measurements were conducted using the Autolab potentiostat (PGSTAT). A square wave potential was applied to the working electrode at $0 \mathrm{~V}$ for $10 \mathrm{~s}$, reducing potential for $90 \mathrm{~s}$ and $0 \mathrm{~V}$ for $20 \mathrm{~s}$. The reducing potentials ranged between 0 to $-0.6 \mathrm{~V}$.

\subsubsection{In-situ electrochemical resonance Raman}

Spectroscopy. The in-situ electrochemical resonance Raman measurements[38] were taken using a Renishaw inVia microscope in a back scattering configuration. The excitation source used a HeNe laser at $633 \mathrm{~nm}$ with $50 \%$ of $12 \mathrm{~mW}$ laser 
power under 50x magnification onto the PEDOT:PSS film on the working electrode. The in-situ Raman measurements were taken when the output current reached steady state during chronoamperometry.

2.3.5 Quartz crystal microbalance. QCM measurements were performed using a Q-sense analyzer (QE401, Biolin Scientific). Swelling measurements were performed as follows. First, we recorded the QCM-D response of the bare $\mathrm{Au}$ sensors in the air, followed by the injection of the 1X PBS solutions into the chamber. This resulted in large shifts in frequency $(f)$ and dissipation of energy $(D)$, due to the density differences between the two media. The measurements were then stopped, the sensors were removed and PEDOT:PSS films with APTES functionalization were fabricated directly on the same sensor as described above. Then, the absolute $f$ value for each polymer coated sensor was obtained both in air and in $1 \mathrm{X}$ PBS, after the $f$ signal was perfectly flat (i.e., $\mathrm{f}<0.5$ $\mathrm{Hz}$ ) assuring that the system is in equilibrium. We then compared the absolute difference in $f$ for multiple overtones between the bare sensor and the polymer coated sensors, both in air and in 1X PBSby using the function "stitched data" of Q-soft software. This function compares the selected datasets based on the raw frequencies measured and excludes the effect of the different densities between the two mediums. Thus, the difference of the $f$ values of the stitched data is directly analogous to the thickness of the polymer in both media, which is calculated by using the Sauerbrey equation below.

$$
\text { eq. 1: } \quad \Delta m=\frac{-17.7}{n} \Delta f_{n}
$$

After the stabilization of QMC-D signals in PBS, water was pumped into the chamber with a flow rate of $70 \mu \mathrm{L} \mathrm{min}{ }^{-1}$ controlled by a peristaltic pump. After a new baseline was established, water-based biotin solution was pumped in the chamber, the flow was stopped and the protein was incubated for $60 \mathrm{~min}$ at a constant temperature of $24{ }^{\circ} \mathrm{C}$. After biotin incubation, water was used to rinse and remove the non-bound protein form the QCM sensors. Next, 1X PBS was pumped in the chamber to establish a new baseline and PBS-based streptavidin solution was pumped in the chamber, the flow was stopped and the protein was incubated for $60 \mathrm{~min}$ at a constant temperature of $24{ }^{\circ} \mathrm{C}$. Finally, PBS was used to rinse and remove the unbound streptavidin from the sensors. To quantify the mass added on each sensor $(\Delta m)$, both Sauerbrey and Young-Voigt viscoelastic models were used. Q-tools, Dfind and Q-soft software were used for the analysis.

2.3.6 Confocal Microscope. An AxioObserver Z1 confocal microscope (Carl Zeiss MicroImaging $\mathrm{GmbH}$ ) was used to detect the binding of Streptavidin Protein, DyLight 405 (control for biotin specificity) and Goat Anti-Mouse IgG,
Alexa Fluor 488 (secondary antibodies selectively binding to the anti-BMP-2 antibodies) on our samples.

\subsubsection{Electrochemical Impedance Spectroscopy.}

Electrochemical Impedance Spectroscopy was adopted to assess surface impedance property modifications after each layer was created. An AUTOLAB PGSTAT100 PotentiostatGalvanostat was employed using $\mathrm{Ag} / \mathrm{AgCl}$ as reference electrode and a platinum wire as counter electrode, in PBS diluted 1:100. The electrochemical area was $500 \times 500 \mu \mathrm{m}$ LxW. The capacitance-frequency plots were constructed by using the complex impedance formula as described in equation 2:

\section{eq. 2: \\ 2.4 OECT fabrication}

OECTs were fabricated via photolithography using a parylene-C lift-off process, as described in [34]. Patterned gold stripes served as source, drain, and gate electrodes. The above reported PEDOT:PSS formulation was used for the active layer and the gate coating, resulting in a device architecture with channels of width-to-length (W/L) of 100/10 $(\mu \mathrm{m} / \mu \mathrm{m})$ and a planar gate of $600 \times 600 \mu \mathrm{m}^{2}$.

\subsection{OECT characterization.}

2.5.1 Output Measurements. Transistor output and transfer characteristics were measured in 1X PBS using a Keithley 2612A with customized LabVIEW software: the potential bias applied at the channel was varied in the range between $0 \mathrm{~V}$ and $-0.6 \mathrm{~V}$, keeping the gate potential fixed, for different $\mathrm{V}_{\mathrm{g}}$ $(0 \mathrm{~V}, 0.1 \mathrm{~V}-0.6 \mathrm{~V})$.

\subsubsection{Transconductance Measurements. Alternating} current (AC) measurements of transconductance versus frequency were run to assess the device performance after each layer was deposited. For the transient (AC) characterization of the OECTs, an NI PXI-4071 digital multimeter was employed to simultaneously measure drain and gate current $\left(I_{d}\right.$ and $\left.I_{g}\right)$. These same measurements were performed as a preliminary quantitative detection for 1.6 $\mathrm{ng} / \mu \mathrm{L}$ of BMP-2 in 0.01X PBS. The semiconducting channel of the OECT was biased at $-0.6 \mathrm{~V}$ and a $0 \mathrm{~V}$ offset and $10 \mathrm{mV}$ amplitude sinusoidal wave was generated from the gate terminal, scanning the frequency range from $1 \mathrm{~Hz}$ to $20 \mathrm{kHz}$. The acquired data were then normalized to the maximum $\mathrm{g}_{\mathrm{m}}$ value. Transconductance cut-off frequencies, taken at $70 \%$ of the curve drop, were extracted for every sample at each subsequent functionalization step and used as layer-dependent figure of merit of the OECT performance. 
All measurements were run on two separate channels but using the same gate; measurements are reported using the standard deviation as error.

\section{Results}

A number of different approaches have been established to impart specificity for biosensing to PEDOT:PSS-based OECTs.[39-41] In this specific case, the streptavidin-biotin chemistry was adopted to functionalize the surface of the device electrodes with the selected antibody for the target analyte.[42,43]

Figure 1 describes our functionalization strategy, which consists of 5 main steps: 1. PEDOT:PSS thin films were plasma-treated with low power intensity to introduce hydroxyl groups on the semiconducting polymer surface without etching it, nor affecting its conductivity; [44] 2. (3Aminopropyl)triethoxysilane (APTES) molecules were covalently bound to the activated surfaces; 3 . biotin units were linked to the APTES-functionalized polymer surfaces; 4. streptavidin molecules bound with high affinity to biotin; 5. a biotinylated, capture antibody (therefore binding to streptavidin) was used to impart specificity towards the target analyte/cytokine.
The plasma treatment induced a reduction of the contact angle of the PEDOT:PSS film from $41.9^{\circ} \pm 0.2^{\circ}$ to $0^{\circ}$, due to the introduction of hydroxyl groups.[44] Plasma-treated surfaces were functionalized with an APTES layer by means of either chemical vapor deposition (CVD) or Soaking (S) process, as described in the Materials and Methods section. Contact angle measurement was used to assess the presence of APTES. In particular, higher values were measured for the CVD samples rather than for the $S$ ones $\left(52^{\circ} \pm 1^{\circ}\right.$ and $46.1^{\circ} \pm 0.4^{\circ}$, respectively). According to [44-46], a hydrophilic surface functionalized with APTES will display values of contact angle close to $45^{\circ}$.

We thoroughly investigated the properties of the two APTES-functionalized PEDOT:PSS films and compared them to those of the pristine ones. Since the presence of APTES would impart dielectric properties to the conducting film, we ran a surface electrical characterization of the deposited APTES layers, using Conductive Atomic Force Microscopy (C-AFM). As expected, C-AFM profiles show a reduction in the surface conductivity when the silane is deposited on top of the PEDOT:PSS thin film (Figure 2a). The drop in the surface conductivity is accompanied by the presence of few isolated conducting spots, which are more abundant for the CVD (Figure 2b) sample than for the S-APTES one. From the

\section{PEDOT:PSS Functionalization Strategy}

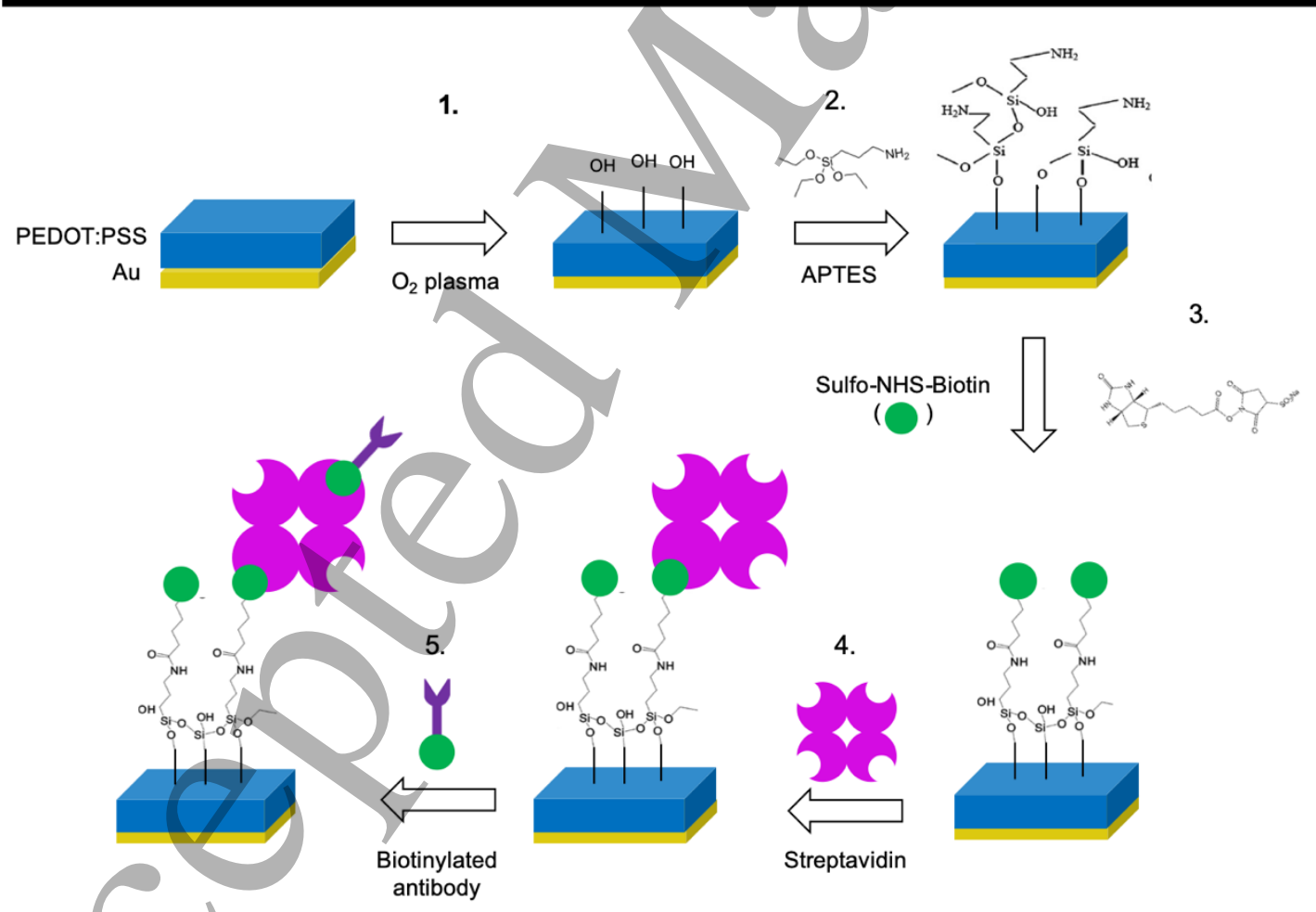

Figure 1: Functionalization strategy employed for PEDOT:PSS coated gold (Au) electrodes. The five sequential steps (1-5) of the established functionalization strategy. 
3 the same scanned areas, we ascribed these spots to defects in the APTES layer, with their high conductivity being due to the PEDOT:PSS layer underneath. From this analysis, it is apparent that a more homogeneous layer was deposited with the soaking process (Figure $2 \mathrm{c}$ ). a)

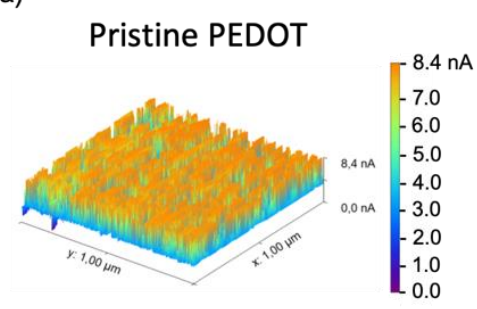

d)

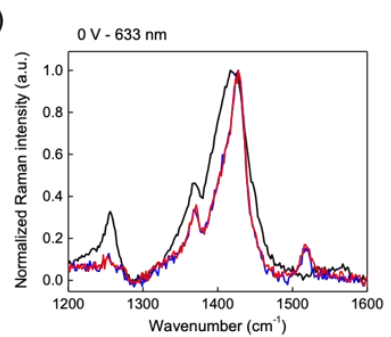

b)

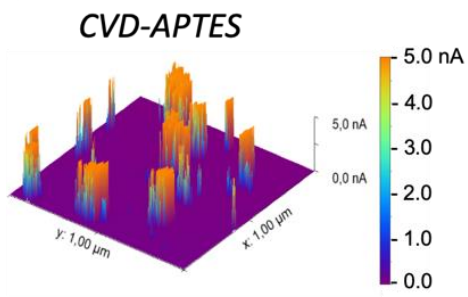

CVD-APTES sample being more difficult to reduce at the same potential as the S-APTES sample.

functionalized PEDOT:PSS films in the reduction regime.

Quartz crystal microbalance with dissipation monitoring e)

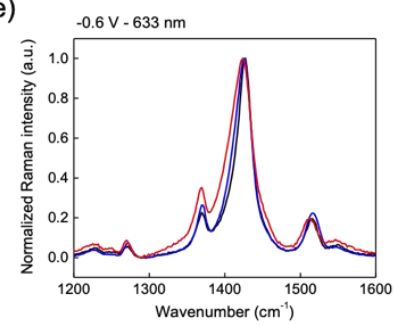

c)

f)

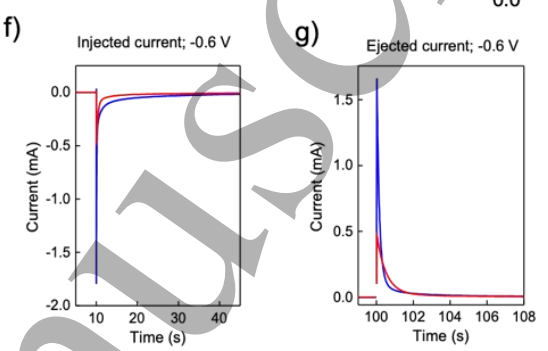

Figure 2: APTES properties and film interaction characterization. Conductive AFM maps of pristine PEDOT:PSS film (a), CVD-APTES (b) and S-APTES (c) functionalized ones. Electrochemical Raman Spectroscopy of pristine PEDOT:PSS film (black), CVD-APTES (red) and S-APTES (blue) functionalized ones, with $0 \mathrm{~V}$ applied on the sample (d) and in the reduction regime (-0.6 V applied) (e). Injected (f) and ejected (g) current in chronoamperometric studies for CVD-APTES (red) and S-APTES (blue) functionalized PEDOT:PSS films in the reduction regime.

Using in-situ electrochemical resonance Raman (QCM-D) analyses were run to understand the effect of the Spectroscopy, we then studied the interaction between silanes on the swelling ability of the PEDOT:PSS thin film in PEDOT:PSS and APTES both in the pristine films and the $\mathrm{NaCl}$ 0.1M.[51] Appropriate controls were used (e.g. gold reduction regime (Fig $2 \mathrm{~d}$ and Fig 2e, respectively). The film, APTES functionalized and pristine PEDOT:PSS films). dedoped PEDOT is resonantly excited at the $633 \mathrm{~nm}$ The QCM-D results (Fig S1) show that the APTES layers excitation wavelength, which provides higher sensitivity to the structural changes during the reduction regime. In/the pristine films (no applied bias), the effect that both APTES deposition approaches have on PEDOT vibrational modes resemble dedoping signatures.[47-49] The Raman spectra show no other molecular structural changes upon the deposition of APTES. As a result of this, APTES silane groups may only interact with PSS-rich regions similar to the crosslinking effect of GOPS on PEDOT:PSS.[50] When CVD- and S-APTES spectra were measured in the reduction regime (-0.6 $\mathrm{V}$ applied to the sample), the latter resulted in spectra closer to those of PEDOT:PSS at the same reduction potential. To further understand this behavior, chronoamperometry measurements were run on both S- and CVD-APTES samples. Lower injected (Fig. 2f) and ejected (Fig. 2g) currents were recorded for the CVD samples, indicative of reduced ion penetration into the film. This in turn leads to less dedoping of the PEDOT:PSS thin film, with the obtained with the two techniques lead to a similar, 5-fold reduction in the film swelling capability (Table 1), compared to the pristine PEDOT:PSS film. Since the same quantitative drop in the film swelling capability is reported for the two deposition processes, the difference between the CVD and SAPTES films might lay in the interaction between the silane layer and PEDOT:PSS in the two different processes. QCM$\mathrm{D}$ was also adopted to investigate the biotin and streptavidin deposition processes. Samples of bare Au, PEDOT:PSS, CVD- and S-APTES were used as substrates and the functionalization was run as described in the Materials and Methods section. Distilled water and PBS were used to run baseline measurements before the incubation with biotin and streptavidin, respectively. As seen from Figure 3, the nonspecific adsorption of biotin onto either Au and PEDOT:PSS is quite low and the excess biotin is easily removed even after the weak rinsing steps performed within the QCM-D flowcell (Table 1). On the contrary, we observe a higher level of biotin 
adsorption for the APTES functionalized samples, with a higher yield for the S-APTES sample $\left(152 \mathrm{ng} / \mathrm{cm}^{2}\right)$ compared

to the CVD-APTES one $\left(23 \mathrm{ng} / \mathrm{cm}^{2}\right)$. The same could be observed for the streptavidin deposition, although in this case higher adsorption levels were measured regardless of the functionalization approach. We hypothesized that physisorption is responsible for the overall high protein levels and that the rinsing steps performed within the QCM-D were not stringent enough to guarantee the removal of the nonspecifically bound molecules. This hypothesis was proved by acquiring confocal microscopy images of samples coated with fluorescently labelled streptavidin in the presence or absence of biotin (Figure S2a). Clearly, fluorescent areas due to the dye-conjugated streptavidin only occur in the presence of biotin. Indeed, the dark area corresponds to the sample where only APTES was present, thus confirming that standard rinsing steps are necessary to remove physiosorbed molecules. Nonetheless, streptavidin levels for the S-APTES/biotin surface reaches the highest value of $292 \mathrm{ng} / \mathrm{cm}^{2}$ (compared to the $212 \mathrm{ng} / \mathrm{cm}^{2}$ for CVD-APTES/biotin ones). This higher, specific functionalization yield supported our choice to proceed with the soaking process in our functionalization strategy for further characterization.

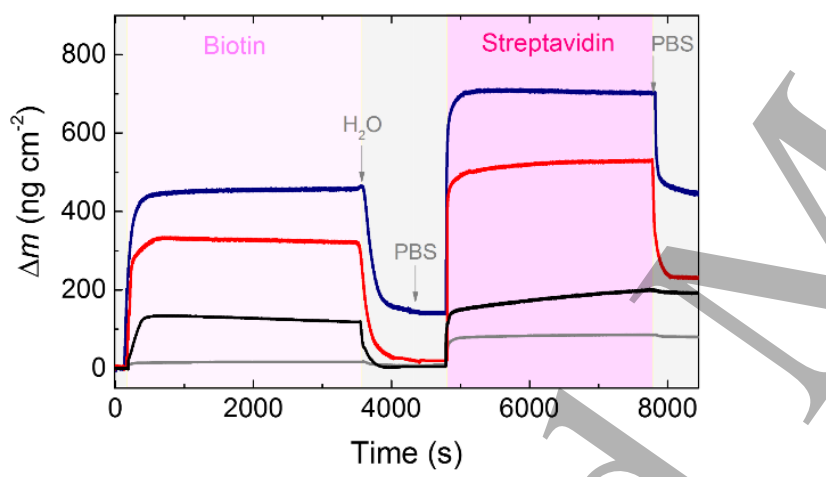

Figure 3: Quartz crystal microbalance with dissipation monitoring (QCM-D) analysis. Biotin and Streptavidin mass adsorption on Au (grey), as well as on PEDOT:PSS (black), CVD- (red) and S- (blue line) APTES functionalized films.

Table 1: Swelling percentage and the amount of biotin and streptavidin adsorbed on APTES functionalized substrate, pristine PEDOT:PSS (P:P) and Au.

\begin{tabular}{c|ccc} 
Substrate & $\begin{array}{c}\text { Swelling } \\
(\%)\end{array}$ & $\begin{array}{c}\text { Bound } \\
\text { biotin } \\
\left(\mathrm{ng} / \mathrm{cm}^{2}\right)\end{array}$ & $\begin{array}{c}\text { Bound } \\
\text { streptavidin } \\
\left(\mathrm{ng} / \mathrm{cm}^{2}\right)\end{array}$ \\
\hline Au & 0 & 2 & 78 \\
Au/P:P & 80 & 2 & 190 \\
Au/P:P/CVD & 15 & 23 & 212 \\
Au/P:P/S & 17 & 152 & 292
\end{tabular}

Once the functionalization unit (APTES + biotin + streptavidin) was deposited, biotinylated capture antibodies, directed towards the analyte of interest (Bone morphogenetic protein 2, BMP-2), were used to impart specificity to our devices. Confocal microscopy was adopted to assess the specificity of the binding for the selected antibody (Figure $\mathrm{S} 2 \mathrm{~b})$.

As shown in figure $4 \mathrm{a}$, we used the same strategy to functionalize the gate of the OECT. Each functionalization layer was characterized by means of electrochemical impedance spectroscopy (EIS) to assess the variations of electrochemical properties (Figure 4b-c, Figure S3 and Figure $\mathrm{S} 4$ ). As shown in figure $\mathrm{S} 4 \mathrm{a}$, the oyerall impedance of the system is increasing with each addition of functionalization layers, indicative of ion barrier formation at the solid-liquid interface. The latter is also verified by the shift of the phase away from $90^{\circ}$ and towards $0^{\circ}$ (Figure S4b). Importantly, the Nyquist plots (Figure 4b) show clear shape differences after the incubation with the proteins, antibodies and, in particular, after BMP-2 addition. The Nyquist plot recorded for the pristine PEDOT:PSS shows low overall impedance with a linear-like behavior indicative of facile ion migration. [52,53] After the proteins are added on the PEDOT:PSS surface, we observed a clear change in the Nyquist plot shape with two distinguished regimes; a semi-circle behavior at high frequencies and a linear behavior with a slope of $\sim 45^{\circ}$ at low frequencies, indicative of delayed diffusion impedance. Importantly, when antibodies and BMP-2 are added, the Nyquist plot shows increase of the overall impedance, with only a large semi-circle shape apparent and absence of the linear regime. This is a strong indication that these bulky molecules, when bound to the electrode surface, impede the ion migration from the electrolyte into the PEDOT:PSS film. The same effect was also visible after the first functionalization step (Figure S3a). These observations are also verified with Figure $4 \mathrm{c}$, which shows capacitance spectra over frequency for the different samples. The capacitance reduces sequentially upon addition of each new layer on top of the semiconducting polymer, especially in the range of frequencies between $100 \mathrm{~Hz}$ and $0.1 \mathrm{~Hz}$, where ionic charging is taking place.[54] This effect can be ascribed to the impaired ion penetration into PEDOT:PSS film due to the addition of each layer acting as ion injection barrier at each functionalization step, similarly to what exerted by adding layers of PLL/PSS on top of the PEDOT:PSS film.[40]

The gate electrode of a PEDOT:PSS-based OECT was functionalized and its performance was monitored after each and every functionalization step, running alternating current (AC) measurements of the OECT transconductance over a range of frequencies $\left(g_{m}(f)\right)$ (Figure $4 a$ and $d$, Figure S5). Transconductance is used as the figure of merit of OECTs and it reflects the gain of the transistor, $\left(g_{m}=\frac{\partial I_{d s}}{\partial V_{g s}}\right)$. OECT configuration and its gate functionalization are reported in the schematic in Figure 4a. Transistor output characteristics were measured before and after gate functionalization with APTES 
(Figure S5), to assess the effect of the APTES deposition on ion penetration. The output curves of an OECT with pristine PEDOT:PSS-coated Au gate electrode are shown in Figure S5a and S5b, and serve as the control. Figure S5c and figure S5d show that the APTES layer deposited by the soaking technique still allows an efficient dedoping of the channel (turning off the device), while no current modulation (absence of dedoping) can be observed in the case of the CVD-treated samples (with I linearly depending on the $\mathrm{V}_{\mathrm{ds}}$ ). Indeed, this can be further proved comparing transistor output characteristics acquired applying different $\mathrm{V}_{\mathrm{gs}}$ (Figures S5ef). As shown, by increasing the applied gate bias $V_{\mathrm{gs}}$ to the $\mathrm{S}$ APTES device, current modulation is achieved, while almost no modulation is observed in the CVD-treated samples.

A drop in the transconductance is observed for every added layer during the functionalization procedure (Figure S6), as a result of the impaired gating.

Normalized $g_{m}(\mathrm{f})$ values for the pristine PEDOT:PSS sample and after the subsequent steps are summarized in drop) were adopted as figure of merit of the device to quantify the influence of each layer on the OECT response (Table 2). The ion penetration limitation, introduced by the additional layers, reduces the transconductance cut-off frequencies.

In particular, the highest drop in the $\mathrm{g}_{\mathrm{m}}$ cut-off is seen between PEDOT:PSS and the addition of the streptavidin layer. The fact that additional layers shift the transconductance curve towards lower frequencies, thus reducing transistor speed, was already seen in previous studies where a layer-bylayer deposition of polystyrene sulfonate and poly-L-lysine (PLL) was adopted as a strategy of device functionalization [40].

Finally, we moved towards a proof of concept experiment to validate our strategy for sensing. BMP-2 is a key mediator for the stem cell differentiation process, being crucial at the earlier stage, since it is involved in increasing mineralization and stimulates osteocalcin mRNA production. [55,56] We therefore decided to use it as a first interesting marker to test our system. a)

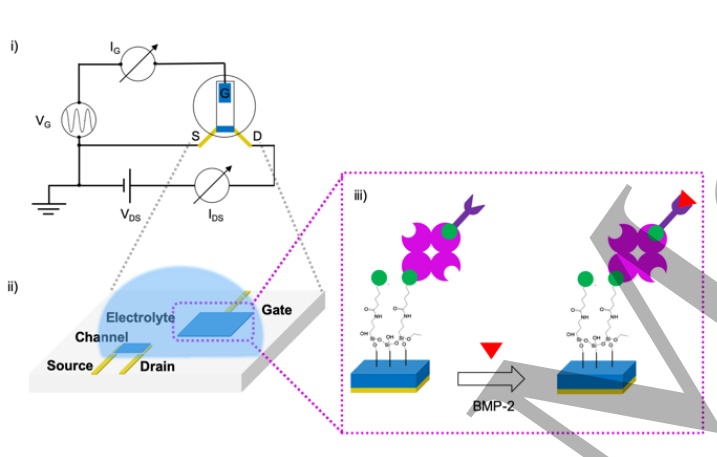

c)

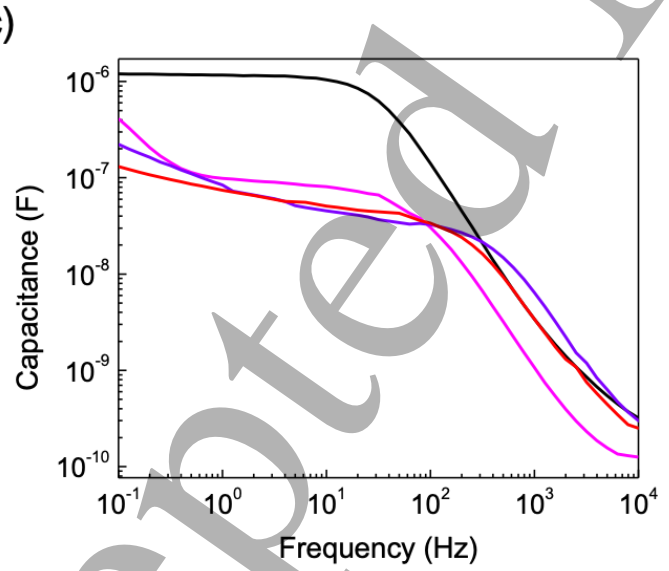

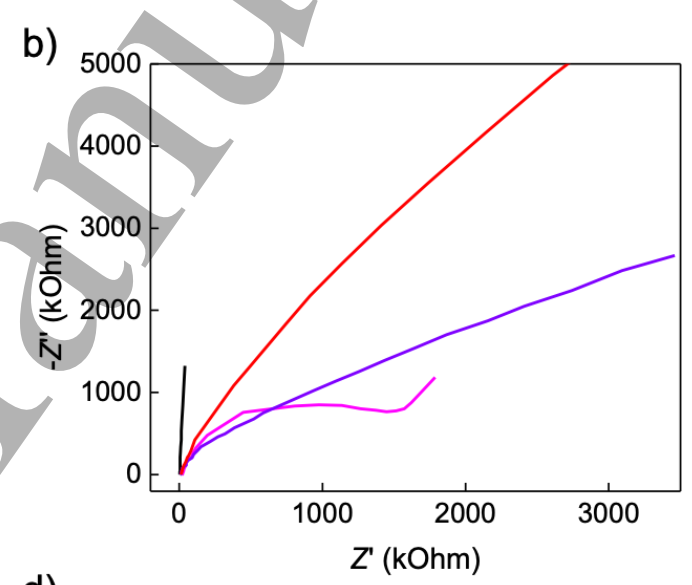

d)

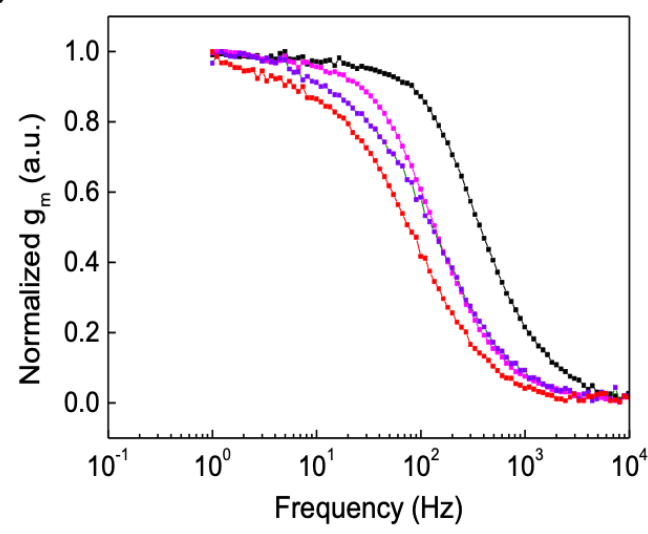

Figure 4: Electrochemical impedance spectroscopy on functionalized electrodes and AC transconductance measurements using OECTs functionalized with anti-BMP-2 antibodies on the PEDOT:PSS gate for BMP2 detection. Schematic of the OECT circuity (i) where t the gate (ii) is functionalized with antibodies against BMP-2 (iii) (a). Nyquist (b) and capacitance vs frequency (c) plots for PEDOT:PSS (black), streptavidin (magenta), antibodies against BMP-2 (violet) and BMP-2 (red). d) Normalized gm vs frequency for PEDOT:PSS (black), streptavidin (magenta), antibodies (violet) and BMP-2 (red).

Figure 4 d. The extracted $g_{m}$ cut-off values $(70 \%$ of the curve 
BMP-2 binding almost halves the device transconductance value, as it passes from $59 \pm 6 \mathrm{~Hz}$ to $36 \pm 2 \mathrm{~Hz}$ (Table 2). This trend is very well aligned with the values of impedance and capacitance acquired for the same samples (Figure 4 b-c), where the highest shifts were reported for streptavidin and BMP-2. This suggests that with the developed functionalization strategy, the PEDOT:PSS based OECT harnesses specificity to selectively detect BMP-2 binding and therefore to monitor stem cells differentiation.

Table 2: Transconductance cut-off values for PEDOT:PSS pristine film and after the addition of streptavidin, anti-BMP2 antibodies (ab) and BMP-2.

\begin{tabular}{l|c} 
Layer & $\mathbf{g}_{\mathbf{m}}$ cut-off $(\mathbf{H z})$ \\
\hline PEDOT:PSS & $200.8 \pm 0.2$ \\
APTES/biotin/streptavidin & $73.9 \pm 0.1$ \\
Anti-BMP-2 ab & $54.8 \pm 0.2$ \\
BMP-2 & $35.0 \pm 2.0$
\end{tabular}

Our results prove that the detection capability of the developed sensor towards BMP-2 holds great promise since the detected value $(1.6 \mathrm{ng} / \mu \mathrm{L})$ is in the order of those used for in vitro stimulation of bone regeneration via soluble osteoinductive factors. [57,58]

\section{Conclusions}

In this work, we have developed a chemical functionalization strategy for PEDOT:PSS-based OECTs to impart specificity towards BMP-2, one of the cytokines released by stem cells undergoing osteogenic differentiation. The approach relies on the subsequent deposition of APTES, biotin, streptavidin and specific capture antibodies to define what we call the 'functionalization unit'. We proved that strong chemical interactions guarantee the specific and selective binding of the target antibodies. The electrical properties of functionalized electrode were assessed at each functionalization step. The soaking process was selected for the APTES deposition, while, the more invasive, chemical vapor deposition was discarded, by impeding ion diffusion at the electrode-electrolyte interface and therefore affecting OECT operation.

We finally demonstrated that the functionalized electrode could be used as gate for OECT biosensors for the detection of BMP-2, a cytokine crucial at the earlier stage of cell osteogenic differentiation, involved in increasing mineralization and osteocalcin production. Antibodies towards this cytokine were used as the last functionalization step and a proof-of-concept detection of BMP-2 binding was run successfully. The $\mathrm{g}_{\mathrm{m}}$ cut-off value was used as quantitative parameter to assess the impact of the functionalization on the device performances. The possibility of detecting BMP-2 levels close to those used in vitro to induce stem cell differentiation makes this functionalization strategy eligible for its use for the detection of cytokine levels in response to external osteogenic stimuli. This work paves the way for realtime, specific sensing of a wide range of cytokines or analytes released during stem cell osteogenic differentiation (e.g. osteocalcin, osteopontin, osteonectin, collagen type I), depending on the selected capture element (e.g. antibodies, aptamers). We foresee that the detection will be performed downstream to the culture chamber, and that sample preparation will proceed the actual measurement. This approach will represent a valuable instrument to provide researchers with further insights and information on cell differentiation processes.

\section{Acknowledgements}

D.I. would like to acknowledge the financial support by the H2020-MSCA-IF-2015 grant, "SMART-BONE" (GA No. 704175). D.I. and F.D. would like to thank Tobias Cramer for the fruitful discussions and insights. A.S. and S. I would like to acknowledge support from KAUST.

\section{References}

[1] Anon International Osteoporosis Foundation

[2] Tu K N, Lie J D, Wan C K V, Cameron M, Austel A G, Nguyen J K, Van K and Hyun D 2018 Osteoporosis: A Review of Treatment Options. P T 43 92-104

Pavone V, Testa G, Giardina S M C, Vescio A, Restivo D A and Sessa G 2017 Pharmacological therapy of osteoporosis: A systematic current review of literature Front. Pharmacol. 8 1-7

[4] Kiernan J, Hu S, Grynpas M D, Davies J E and Stanford W L 2016 Systemic Mesenchymal Stromal Cell Transplantation Prevents Functional Bone Loss in a Mouse Model of Age-Related Osteoporosis Stem Cells Transl. Med. 5 683-693

[5] Saito A, Nagaishi K, Iba K, Mizue Y, Chikenji T, Otani M, Nakano M, Oyama K, Yamashita T and Fujimiya M 2018 Umbilical cord extracts improve osteoporotic abnormalities of bone marrow-derived mesenchymal stem cells and promote their therapeutic effects on ovariectomised rats Sci. Rep. 8 1-16

[6] Bae S J, Kim H J, Won H Y, Min Y K and Hwang E S 2017 Acceleration of osteoblast differentiation by a novel osteogenic compound, DMP-PYT, through activation of both the BMP and Wnt pathways Sci. Rep. 7 1-10

[7] Persson M, Lehenkari P P, Berglin L, Turunen S, Finnilä M A J, Risteli J, Skrifvars M and Tuukkanen J 2018 Osteogenic Differentiation of Human Mesenchymal Stem 


\section{cells in a 3D Woven Scaffold Sci. Rep. 8 1-12}

[8] Iandolo D, Ravichandran A, Liu X, Wen F, Chan J K Y, Berggren M, Teoh S H and Simon D T 2016 Development and Characterization of Organic Electronic Scaffolds for Bone Tissue Engineering Adv. Healthc. Mater. 5 1505-12

[9] Llopis-Hernández V, Cantini M, González-García C, Cheng Z A, Yang J, Tsimbouri P M, García A J, Dalby M J and Salmerón-Sánchez M 2016 Material-driven fibronectin assembly for high-efficiency presentation of growth factors Sci. Adv. 2 e1600188

[10] Juignet L, Charbonnier B, Dumas V, Bouleftour W, Thomas M, Laurent C, Vico L, Douard N, Marchat D and Malaval L 2017 Macrotopographic closure promotes tissue growth and osteogenesis in vitro Acta Biomater. 53 536-48

[11] Guex A G, Puetzer J L, Armgarth A, Littmann E, Stavrinidou E, Giannelis E P, Malliaras G G and Stevens M M 2017 Highly porous scaffolds of PEDOT:PSS for bone tissue engineering Acta Biomater. 62 91-101

[12] Zhang K, Wang S, Zhou C, Cheng L, Gao X, Xie X, Sun J, Wang H, Weir M D, Reynolds M A, Zhang N, Bai Y and Xu H H K 2018 Advanced smart biomaterials and constructs for hard tissue engineering and regeneration Bone Res. 6

[13] Rutkovskiy A, Stensløkken K-O and Vaage I J 2016 Osteoblast Differentiation at a Glance Med. Sci. Monit. Basic Res. 22 95-106

[14] Long F 2012 Building strong bones: Molecular regulation of the osteoblast lineage Nat. Rev. Mol. Cell Biol. 13 27-38

[15] Robertson S N, Campsie P, Childs P G, Madsen F, Donnelly H, Henriquez F L, Mackay W G, SalmerónSánchez M, Tsimbouri M P, Williams C, Reid S and Dalby M J 2018 Control of cell behaviour through nanovibrational stimulation: nanokicking Philos. Trans. $R$ Soc. A Math. Phys. Eng. Sci. 37620170290

[16] Tandon B, Blaker J J and Cartmell S H 2018 Piezoelectric materials as stimulatory biomedical materials and scaffolds for bone repair Acta Biomater. 73 1-20

[17] Shin S R, Kilic T, Zhang Y S, Avci H, Hu N, Kim D, Branco C, Aleman J, Massa S, Silvestri A, Kang J, Desalvo A, Hussaini M A, Chae S K, Polini A, Bhise N, Hussain M A, Lee H Y, Dokmeci M R and Khademhosseini A 2017 Label-Free and Regenerative Electrochemical Microfluidic Biosensors for Continual Monitoring of Cell Secretomes Adv. Sci. 4 1-14

[18] Pitsalidis C, Ferro M P, Iandolo D, Tzounis L, Inal S and Owens R M 2018 Transistor in a tube: A route to threedimensional bioelectronics Sci. Adv. 4 /eaat4253

[19] Song J H, Lee S M and Yoo K H 2018 Label-free and real-time monitoring of human mesenchymal stem cell differentiation in 2D and 3D cell culture systems using impedance cell sensors RSC Adv. 8 31246-54

[20] Rivnay J, Owens R M and Malliaras G G 2014 The rise of organic bioelectronics Chem. Mater. 26 679-85
Fidanovski K and Mawad D 2019 Conjugated Polymers in Bioelectronics: Addressing the Interface Challenge $A d v$. Healthc. Mater. 8 1-9

[22] Decataldo F, Cramer T, Martelli D, Gualandi I, Korim W S, Yao S T, Tessarolo M, Murgia M, Scavetta E, Amici R and Fraboni B 2019 Stretchable Low Impedance Electrodes for Bioelectronic Recording from Small Peripheral Nerves Sci. Rep. 910598

[23] Keene S T, Marais A, Curto V F, Salleo A and Parlak O 2018 Molecularly selective nanoporous membrane-based wearable organic electrochemical device for noninvasive cortisol sensing Sci. Adv. 4 eaar2904

[24] Decataldo F, Barbalinardo M, Tessarolo M, Vurro V, Calienni M, Gentili D, Valle F, Cavallini M and Fraboni B 2019 Organic Electrochemical Transistors: Smart Devices for Real-Time Monitoring of Cellular Vitality Adv. Mater. Technol. 1900207

[25] Gualandi I, Marzocchi M, Achilli A, Cavedale D, Bonfiglio A and Fraboni B 2016 Textile Organic Electrochemical Transistors as a Platform for Wearable Biosensors Sci. Rep. 633637

[26] Pani D, Dessi A, Saenz-Cogollo J F, Barabino G, Fraboni B and Bonfiglio A 2016 Fully Textile, PEDOT:PSS Based Electrodes for Wearable ECG Monitoring Systems IEEE Trans. Biomed. Eng. 63 540-9

[27] Bonisoli A, Marino A, Ciofani G and Greco F 2017 Topographical and Electrical Stimulation of Neuronal Cells through Microwrinkled Conducting Polymer Biointerfaces Macromol. Biosci. 17 1-8

[28] Kim S M, Kim N, Kim Y, Baik M S, Yoo M, Kim D, Lee W J, Kang D H, Kim S, Lee K and Yoon M H 2018 Highperformance, polymer-based direct cellular interfaces for electrical stimulation and recording NPG Asia Mater. 10 $255-65$

[29] Marino A, Barsotti J, De Vito G, Filippeschi C, Mazzolai B, Piazza V, Labardi M, Mattoli V and Ciofani G 2015 Two-Photon Lithography of 3D Nanocomposite Piezoelectric Scaffolds for Cell Stimulation ACS Appl. Mater. Interfaces 7 25574-9

[30] Hardy J G, Sukhavasi R C, Aguilar D, Villancio-Wolter M K, Mouser D J, Geissler S A, Nguy L, Chow J K, Kaplan D L and Schmidt C E 2015 Electrical stimulation of human mesenchymal stem cells on biomineralized conducting polymers enhances their differentiation towards osteogenic outcomes J. Mater. Chem. B 3 8059-64

[31] Rivnay J, Inal S, Salleo A, Owens R M, Berggren M and Malliaras G G 2018 Organic electrochemical transistors Nat. Rev. Mater. 317086

[32] Inal S, Malliaras G G and Rivnay J 2017 Benchmarking organic mixed conductors for transistors Nat. Commun. 8 1767

[33] Braendlein M, Pappa A M, Ferro M, Lopresti A, Acquaviva C, Mamessier E, Malliaras G G and Owens R M 2017 Lactate Detection in Tumor Cell Cultures Using Organic Transistor Circuits Adv. Mater. 29 
[34] Pappa A-M, Curto V F, Braendlein M, Strakosas X, Donahue M J, Fiocchi M, Malliaras G G and Owens R M 2016 Organic Transistor Arrays Integrated with FingerPowered Microfluidics for Multianalyte Saliva Testing Adv. Healthc. Mater. 5 2295-302

[35] Kim D J, Lee N E, Park J S, Park I J, Kim J G and Cho H J 2010 Organic electrochemical transistor based immunosensor for prostate specific antigen (PSA) detection using gold nanoparticles for signal amplification Biosens. Bioelectron. 25 2477-82

[36] Fu Y, Wang N, Yang A, Law H K wai, Li L and Yan F 2017 Highly Sensitive Detection of Protein Biomarkers with Organic Electrochemical Transistors Adv. Mater. 29

[37] Fiorilli S, Rivolo P, Descrovi E, Ricciardi C, Pasquardini L, Lunelli L, Vanzetti L, Pederzolli C, Onida B and Garrone E 2008 Vapor-phase self-assembled monolayers of aminosilane on plasma-activated silicon substrates $J$. Colloid Interface Sci. 321 235-41

[38] Nightingale J, Wade J, Moia D, Nelson J and Kim J S 2018 Impact of Molecular Order on Polaron Formation in Conjugated Polymers J. Phys. Chem. C 122 29129-40

[39] Berezhetska O, Liberelle B, De Crescenzo G and Cicoira F 2015 A simple approach for protein covalent grafting on conducting polymer films J. Mater. Chem. B 3 5087-94

[40] Pappa A-M, Inal S, Roy K, Zhang Y, Pitsalidis C, Hama A, Pas J, Malliaras G G and Owens R M 2017 Polyelectrolyte Layer-by-Layer Assembly on Organic Electrochemical Transistors ACS Appl. Mater. Interfaces 9 $10427-34$

[41] Strakosas X, Sessolo M, Hama A, Rivnay J, Stavrinidou E, Malliaras G G and Owens R M 2014 A facile biofunctionalisation route for solution processable conducting polymer devices J. Mater. Chem. B 2 2537-45

[42] DeChancie J and Houk K N 2007 The Origins of Femtomolar Protein-Ligand Binding: Hydrogen Bond Cooperativity and Desolvation Energetics in the Biotin(Strept)Avidin Binding Site J Am Chem Soc 129 5419-29

[43] Chivers C E, Crozat E, Chu C, Moy V T, Sherratt D J and Howarth M 2010 A streptavidin variant with slower biotin dissociation and increased mechanostability Nat. Methods 7 391-3

[44] Ohayon D, Pitsalidis C, Pappa A M, Hama A, Zhang Y, Gallais L and Owens R M 2017 Laser Patterning of SelfAssembled Monolayers on PEDOT:PSS Films for Controlled Cell Adhesion Adv. Mater. Interfaces 4

[45] Zeng X, Xu G, Gao Y and An Y 2011 Surface Wettability of (3-Aminopropyl)triethoxysilane Self-Assembled Monolayers J. Phys. Chem. B 115 450-4

[46] Zhang F, Sautter K, Larsen A M, Findley D A, Davis R C, Samha H and Linford M R 2010 Chemical Vapor Deposition of Three Aminosilanes on Silicon Dioxide: Surface Characterization, Stability, Effects of Silane Concentration, and Cyanine Dye Adsorption

[47] Garreau S, G. Louarn, J. P. Buisson, G. Froyer and S.
Lefrant 1999 In situ spectroelectrochemical Raman studies of poly(3,4-ethylenedioxythiophene) (PEDT) Macromolecules 32 6807-12

[48] Łapkowski M and Pron A 2000 1-s2.0S0379677999002714-main.pdf 79-83

[49] Chia P J, Chua L L, Sivaramakrishnan S, Zhuo J M, Zhao L H, Sim W S, Yeo Y C and Ho P K H 2007 Injectioninduced de-doping in a conducting polymer during device operation: Asymmetry in the hole injection and extraction rates Adv. Mater. 19 4202-7

[50] Håkansson A, Han S, Wang S, Lu J, Braun S, Fahlman M, Berggren M, Crispin X and Fabiano S 2017 Effect of (3glycidyloxypropyl)trimethoxysilane (GOPS) on the electrical properties of PEDOT:PSS films J. Polym. Sci. Part B Polym. Phys. 55 814-20

[51] Savva A, Wustoni S and Inal S 2018 Ionic-to-electronic coupling efficiency in PEDOT:PSS films operated in aqueous electrolytes J. Mater. Chem. C 6 12023-30

[52] D. Pletcher, R. Greff, R. Peat, L. Peter, J. Robinson 2001 Instrumental Methods in Electrochemistry (Cambridge, UK: Woodhead Publishing)

[53] A. J. Bard, L. R. Faulkner, J. Leddy C G Z 1980 Electrochemical Methods: Fundamentals and Applications (New York: Wiley)

[54] Savva A, Cendra C, Giugni A, Torre B, Surgailis J, Ohayon D, Giovannitti A, McCulloch I, Di Fabrizio E, Salleo A, Rivnay J and Inal S 2019 Influence of Water on the Performance of Organic Electrochemical Transistors Chem. Mater. 31 927-37 Wozney J M 2002 Overview of Bone Morphogenetic Proteins Spine (Phila. Pa. 1976). 27 S2-8

[56] Duplomb L, Dagouassat M, Jourdon P and Heymann D 2006 Concise Review: Embryonic Stem Cells: A New Tool to Study Osteoblast and Osteoclast Differentiation Stem Cells 25 544-52

[57] Kim J, Kim I S, Cho T H, Lee K B, Hwang S J, Tae G, Noh I, Lee S H, Park Y and Sun K 2007 Bone regeneration using hyaluronic acid-based hydrogel with bone morphogenic protein-2 and human mesenchymal stem cells Biomaterials 28 1830-7

[58] Park S H, Kwon J S, Lee B S, Park J H, Lee B K, Yun J H, Lee B Y, Kim J H, Min B H, Yoo T H and Kim M S 2017 BMP2-modified injectable hydrogel for osteogenic differentiation of human periodontal ligament stem cells Sci. Rep. 7 1-15 